\title{
TISSUE-ENGINEERED VALVED CONDUITS IN THE PULMONARY CIRCULATION
}

Ulrich A. Stock, MD

Mitsugi Nagashima, MD $^{\text {a }}$

Philipe N. Khalil, MD

Georg D. Nollert, MD

Tanja Herden ${ }^{\mathrm{a}}$

Jason S. Sperling, MD

Adrian Moran, $\mathrm{MD}^{\mathrm{c}}$

Jamie Lien, BA ${ }^{\mathrm{f}}$

David P. Martin, $\mathrm{PhD}^{\mathrm{e}}$

Frederick J. Schoen, $\mathrm{MD}, \mathrm{PhD}^{\mathrm{d}}$

Joseph P. Vacanti, MD

John E. Mayer, Jr, MD
Objective: Bioprosthetic and mechanical valves and valved conduits are unable to grow, repair, or remodel. In an attempt to overcome these shortcomings, we have evaluated the feasibility of creating 3-leaflet, valved, pulmonary conduits from autologous ovine vascular cells and biodegradable polymers with tissue-engineering techniques.

Methods: Endothelial cells and vascular medial cells were harvested from ovine carotid arteries. Composite scaffolds of polyglycolic acid and polyhydroxyoctanoates were formed into a conduit, and 3 leaflets (polyhydroxyoctanoates) were sewn into the conduit. These constructs were seeded with autologous medial cells on 4 consecutive days and coated once with autologous endothelial cells. Thirty-one days $( \pm 3$ days) after cell harvesting, 8 seeded and 1 unseeded control constructs were implanted to replace the pulmonary valve and main pulmonary artery on cardiopulmonary bypass. No postoperative anticoagulation was given. Valve function was assessed by means of echocardiography. The constructs were explanted after 1, 2, 4, 6, 8, 12,16 , and 24 weeks and evaluated macroscopically, histologically, and biochemically.

Results: Postoperative echocardiography of the seeded constructs demonstrated no thrombus formation with mild, nonprogressive, valvular regurgitation up to 24 weeks after implantation. Histologic examination showed organized and viable tissue without thrombus. Biochemical assays revealed increasing cellular and extracellular matrix contents. The unseeded construct developed thrombus formation on all 3 leaflets after 4 weeks.

Conclusion: This experimental study showed that valved conduits constructed from autologous cells and biodegradable matrix can function in the pulmonary circulation. The progressive cellular and extracellular matrix formation indicates that the remodeling of the tissue-engineered structure continues for at least 6 months. (J Thorac Cardiovasc Surg 2000;119:732-40) $\mathrm{n}$ recently published studies our laboratory has been able to replace successfully a single pulmonary valve leaflet ${ }^{1}$ or segments of the main pulmonary artery ${ }^{2}$ by using tissue-engineering principles (Fig 1). These experiments have been conducted by using a biodegradable scaffold of polyglycolic acid (PGA) and polylactic acid (PLA) seeded with autologous vascular cells.

From the Department of Cardiovascular Surgery, ${ }^{a}$ Department of Surgery, ${ }^{\mathrm{b}}$ Department of Cardiology, ${ }^{\mathrm{c}}$ Children's Hospital, Boston, Mass; the Department of Pathology, ${ }^{\mathrm{d}}$ Brigham and Women's Hospital, Boston, Mass; Metabolix Inc, ${ }^{e}$ Cambridge, Mass; and the Department of Chemical Engineering, ${ }^{f}$ Massachusetts Institute of Technology, Cambridge, Mass.

Supported by grants from the Department of Cardiac Surgery, Children's Hospital, Boston, Mass; Deutsche Forschungsgemeinschaft (Sto 359/1-1); and the National Institutes of Health (HL-97-005).

Read at the Seventy-ninth Annual Meeting of The American Association for Thoracic Surgery, New Orleans, La, April 18-21, 1999.

Received for publication April 22, 1999; revisions requested Sept 15,
Despite these encouraging results, attempts to replace all 3 pulmonary valve leaflets were unsatisfactory because of the relatively high initial rigidity of the PGA-PLA scaffold tissue-engineered leaflets.

In this study we tested a new biodegradable, bioresorbable, and thermoplastic polymer, polyhydroxyoctanoate (PHO), seeded with autologous vascular cells in an

1999; revisions received Nov 19, 1999; accepted for publication Nov 23, 1999.

Address for reprints: John E. Mayer, Jr, MD, Department of Cardiovascular Surgery, Children's Hospital Harvard Medical School, 300 Longwood Ave, Boston, MA 02115 (E-mail: mayer@a1.tch-harvard.edu).

Copyright (C) 2000 by The American Association for Thoracic Surgery.

$0022-5223 / 2000 \$ 12.00+0 \quad \mathbf{1 2 / 6 / 1 0 4 5 8 4}$

doi:10.1067/mtc.2000.104584 


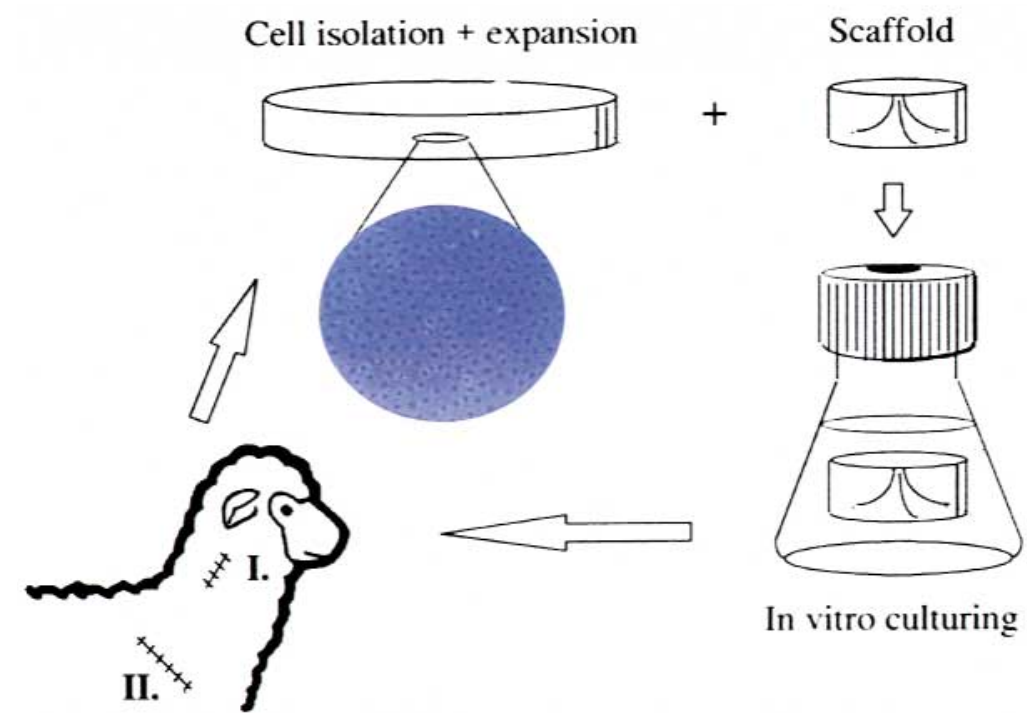

Fig 1. Concept of tissue engineering. Initial vessel harvest (step I) is followed by cell isolation and culture and seeding of the scaffold with further in vitro culturing. The seeded scaffold is implanted back into the same animal (step II).

initial feasibility study for the creation of a 3-leaflet heart valve in the supravalvular pulmonary valve position.

\section{Materials and methods}

The general approach to cell isolation, culture, sorting, and seeding was described in detail previously. ${ }^{3}$ Several modifications have been made in these experiments.

Cell isolation and culture. Segments of the common carotid artery $(2-3 \mathrm{~cm})$ were harvested from 6-week-old Dover lambs $(n=8)$. Endothelial cells were obtained by using a collagenase instillation technique for 20 minutes at $37^{\circ} \mathrm{C}$ at $95 \%$ oxygen and $5 \%$ carbon dioxide $(0.2 \%$ collagenase type A [Boehringer Mannheim] in 1\% bovine serum albumin [Sigma]) and cultured in gelatin precoated ( $1 \%$ gelatin [Sigma]) tissue culture flasks (Corning Inc) by using Medium 199 (Gibco) supplemented with $10 \%$ fetal bovine serum (Sigma), 1\% L-glutamine, penicillin, streptomycin (Sigma), and $50 \mathrm{IU} / \mathrm{mL}$ heparin (Promega). The media was changed either every 3 days or during passaging.

The remaining de-endothelialized vessel segments were minced into 1-mm ${ }^{2}$ pieces and cultured in Dulbecco's modified Eagle's medium high glucose (Gibco) supplemented with $10 \%$ fetal bovine serum, $1 \%$ L-glutamine, penicillin, and streptomycin on P100 dishes (Corning). After migration of medial cells onto the dishes (7-10 days after the harvest), the cells were serially passaged on $1 \%$ collagen-precoated tissueculture ware (Corning). Approximately 14 additional days were needed to obtain 12 confluent T75 tissue-culture flasks (Corning).

Biodegradable polymer and conduit design. The $\mathrm{PHO}$ was provided from Metabolix Inc, the PGA was purchased from Smith and Nephew Inc, and polydioxanone was purchased from Ethicon, Inc.

The conduit (20 $\mathrm{mm}$ in length and $18 \mathrm{~mm}$ in internal diameter) was composed of nonporous PHO film $(240-\mu \mathrm{m}$ thick) with layers of PGA felt (1-mm thickness) on the inside and outside of the PHO. The leaflets consisted of a monolayer of porous PHO (120- $\mu \mathrm{m}$ thickness). A salt-leaching technique ${ }^{4}$ was used to create a porous material (pore sizes of $80-180$ $\mu \mathrm{m}$; Fig $2, A$ ) for the leaflets. The leaflets were sutured to the conduit wall by using 6-0 polydioxanone running sutures (Fig 2, B). After completion of the suturing, the conduits were immersed for 24 hours in $2 \%$ polyvinyl alcohol (Sigma) to decrease hydrophobic surface characteristics and increase the wettability of the PHO. After air drying, the constructs were sterilized with cold ethylene oxide.

Cell seeding. After 23 days ( \pm 3 days) in cell culture, an average of $17.38 \times 10^{6}\left( \pm 0.9 \times 10^{6}\right)$ medial cells were dripped onto the construct on each of 4 consecutive days. Finally, $14.85 \times 10^{6}\left( \pm 0.25 \times 10^{6}\right)$ endothelial cells were seeded once on the inside of the construct. The polymer constructs were precoated before the seeding with laminin $(5 \mu \mathrm{g} / \mathrm{mL}$ [Sigma] for 24 hours to improve cell-polymer attachment. The constructs were cultured in Dulbecco's modified Eagle's medium high glucose supplemented with $10 \%$ fetal bovine serum, $1 \%$ L-glutamine, penicillin, and streptomycin in tissue-culture plastic ware.

After 1 final incubation day in an Erlenmeyer flask (Corning) with culture media, the constructs were implanted.

Implantation. At 31 days ( \pm 3 days) after the initial cell harvest, the same 8 animals from which the initial vessel was harvested (average weight, $25 \pm 5 \mathrm{~kg}$ ) underwent replace- 

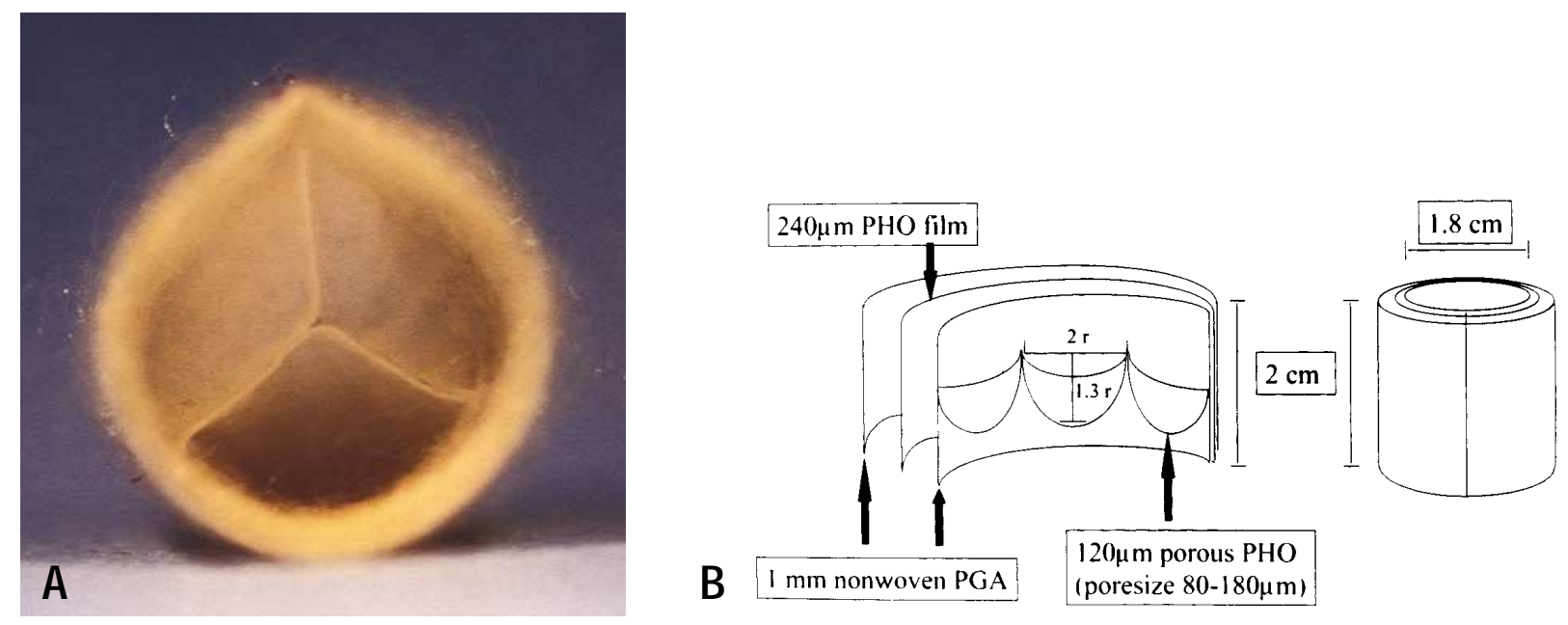

Fig 2. A, Polymer scaffold (frontal view) $18 \mathrm{~mm}$ in diameter and $20 \mathrm{~mm}$ in length. A $240-\mu \mathrm{m}$ nonporous PHO conduit wall with 1-mm nonwoven PGA felts on the inside and outside was used. Three leaflets of porous PHO were sutured into the conduit. B, Sketch of scaffold design.

ment of the pulmonary valve and main pulmonary artery with an autologous tissue-engineered valved conduit. In one other animal an acellular polymer valved conduit was implanted. Anesthesia was induced with $2 \mathrm{mg} / \mathrm{kg}$ ketamine, $0.02 \mathrm{mg} / \mathrm{kg}$ atropine, and an intravenous bolus infusion of 2 $\mathrm{mg} / \mathrm{kg}$ propofol. Anesthesia was maintained by use of inhalational isofluorane. The heart was exposed by a left anterolateral thoracotomy entering the chest through the 4th intercostal space. Systemic anticoagulation was induced with $400 \mathrm{IU}$ of heparin per kilogram. By means of femoral arterial and right atrial venous cannulation, normothermic cardiopulmonary bypass was established. On bypass, 0.01 $\mathrm{mg} / \mathrm{kg}$ fentanyl and $0.02 \mathrm{mg} / \mathrm{kg}$ pancuronium were administered to ensure anesthesia. With the heart beating, the pulmonary artery was transected, and a segment of the main pulmonary artery and all 3 native leaflets were removed. The valved conduit was implanted by using running 5-0 monofilament sutures (Prolene, Ethicon, Inc). Heparin was reversed with 300 IU of protamine per kilogram after weaning from bypass. The thoracic wall was closed in layers by using resorbable sutures, and an intercostal nerve block with $0.25 \%$ bupivacaine was administered. No further anticoagulation was given. All animals received $1000 \mathrm{mg}$ of cefazolin (Apothecon) for the first postoperative week on a daily basis. For pain control, intramuscular buprenorphin injections were given for the first 3 days and thereafter as necessary. All animals received humane care in compliance with the "Guide for the Care and Use of Laboratory Animals" published by the National Institutes of Health (National Institutes of Health publication No. 85-23, revised 1985). After 7 days in Children's Hospital research facilities, the animals were moved to an off-site indoor housing facility.

Evaluation of the tissue-engineered tricuspid conduits. After implantation, Doppler echocardiography with either an Accuson 128 or Hewlett-Packard Sonos 1500 Cardiac Imager equipped with a 7- to 7.5-MHz phased-arrayed transducer was used periodically to evaluate valve function. Two-dimensional echocardiographic cDoppler examination was performed of the right ventricular outflow tract, conduit, and distal main pulmonary artery immediately before chest closure. Subsequent evaluations after $1,2,4,6,8,12$, and 24 weeks included imaging of the conduit and leaflets from a long- and short-axis view. Qualitative evaluation of pulmonary valve competence was made by using color flow Doppler mapping. The explanted conduit wall and the leaflets were evaluated macroscopically and histologically. For determination of cellular and extracellular components of the conduit wall, histochemical assays were performed. DNA content was measured by using a commercially available cell proliferation assay kit (CyQuant, Molecular Probes Inc). For determination of collagen content, tissue 4-hydroxyproline levels were determined. ${ }^{5}$ Elastin was quantified after tissue extraction by using $0.1 \%$ hot oxalic acid with a FASTIN elastin assay (Biocolor Ltd) and proteoglycan-glycosaminoglycan content after tissue extraction with guanidine $\mathrm{HCl}$ with a BLYSCAN proteoglycan-glycosaminoglycan assay (Biocolor Ltd).

Molecular weight of the PHO material after explantation was determined by using gel-permeation chromatography. Isolated polymers were dissolved in chloroform at approximately 1 $\mathrm{mg} / \mathrm{mL}$, and samples $(50 \mu \mathrm{L})$ were evaluated by chromatography on a Waters Stryagel HT6E column at a flow rate of $1 \mathrm{~mL}$ of chloroform per minute at room temperature by using a refractive index detector. Molecular masses were determined relative to polystyrene standards of narrow polydispersity.

\section{Results}

All animals survived the operative procedure. Doppler echocardiography revealed no signs of thrombus formation on the conduit wall or on the leaflets of 

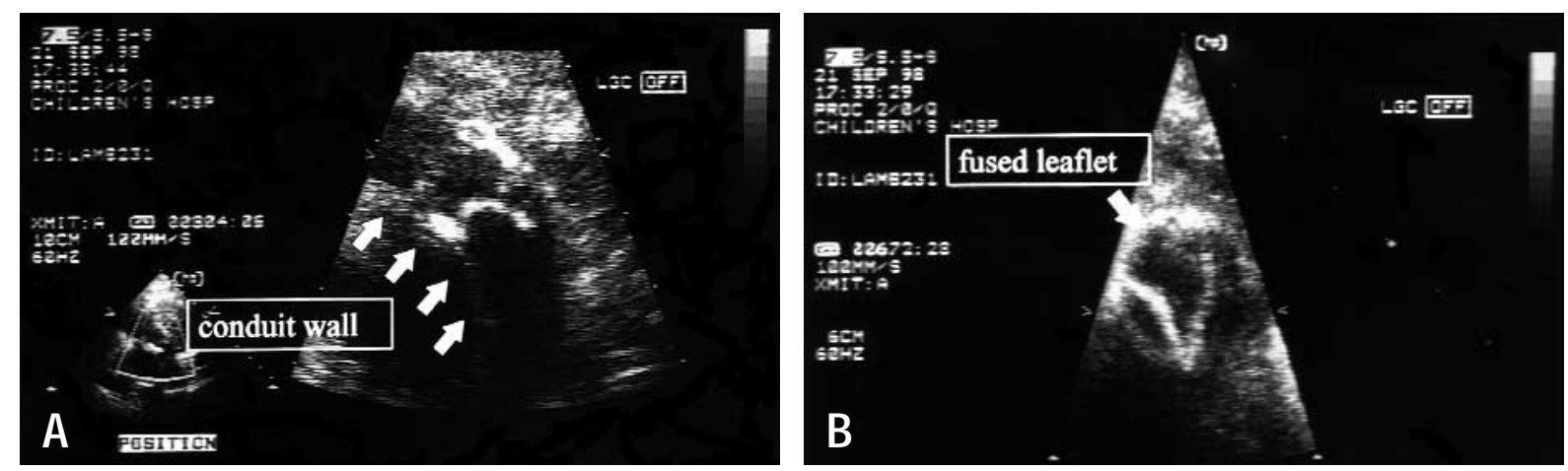

Fig 3. Echocardiographic assessment. A, Sixteen weeks after implantation: long axis. B, Sixteen weeks after implantation: short axis. One leaflet was fused with the conduit wall.

the cellular conduits (Fig 3, $A$ and $B$ ). In two animals the echocardiography results suggested one nonfunctioning leaflet with questionable attachment to the conduit wall. Increasing scar tissue formation and the overlying lung around the tissue-engineered conduit eventually limited the echocardiographic window in all cases.

The macroscopic evaluation of the seeded conduits showed no signs of thrombus formation with clear evidence of tissue formation and a shiny surface of the leaflets and the conduit wall. In each of the two animals with suspicious echocardiographic results, one leaflet was found to be fused to the conduit wall. At 24 weeks after implantation, the conduit showed a marked thickening of the conduit wall (Fig 4, $A$ and $B$ ). In none of the conduits was an increase in diameter observed. The unseeded control construct showed after 4 weeks of in vivo severe thrombus formation on all 3 leaflets (Fig 4,C).

The histologic examination of the conduit wall showed laminated fibrous tissue uniformly covering the surface. Transmural cords of vascularized tissue-filled interstices were created by partial degradation of the polymer. Flattened cells, resembling endothelial cells, covered the luminal surface of the conduit wall and extended onto the proximal leaflets. Immunohistochemical staining with human von Willebrand factor confirmed this observation. Healing was less complete on the leaflets than on the conduit wall. A Movat pentachrome stain, which stains elastin black, collagen yellow, and glycosaminoglycans blue-green, showed significant amounts of stainable collagen and proteoglycans but no stainable elastin. There was essentially no thrombus formation and a mild foreign body response to the polymer (Fig 5, A-C).

The biochemical assays revealed the following results. The DNA content of the conduit wall progressively increased to reach $62 \%$ of the native pulmonary artery after 6 months, indicating gradually increasing cellulari- ty (Fig 6, A). The 4-hydroxyproline content as a measure of collagen content increased over the observed time and reached a maximum of $460 \%$ of the native pulmonary artery after 6 months (Fig 6, B). The elastin assay revealed a steady increase with a maximum content of $215 \%$ of the native pulmonary artery after 16 weeks (Fig $6, C)$. The determination of proteoglycans and glycosaminoglycans in the explanted conduits showed a continuing production of these extracellular matrix components with a maximum content exceeding the native pulmonary artery by $180 \%$ (Fig 6, D).

Although histologic examination after 6 weeks did not show any significant PGA, PHO material was still evident in the conduit and the leaflets at 24 weeks. Analysis of the residual PHO indicated a molecular weight loss of $26 \%$, suggesting a much longer degradation profile for PHO than PGA (Fig 7).

\section{Discussion}

Prosthetic heart valves are durable and have good flow dynamics, but they have well-known limitations, including thrombogenicity, susceptibility to infection, and lack of growth potential. Homograft valves are less thrombogenic and may be more resistant to infection but lack growth potential and are less durable than prosthetic valves. ${ }^{6}$ In the pediatric population the incidence of calcification of homografts is high as well. ${ }^{7}$ None of the currently used heart valve devices meet the criteria for an ideal valve replacement outlined by Harken and Curtis ${ }^{8}$ many years ago (Table I).

These limitations of currently available prosthetic and bioprosthetic valves and conduit arteries have led us to investigate the tissue-engineering approach to constructing valves and arteries.

Tissue engineering is an interdisciplinary field that applies the principles of engineering and the life sci- 

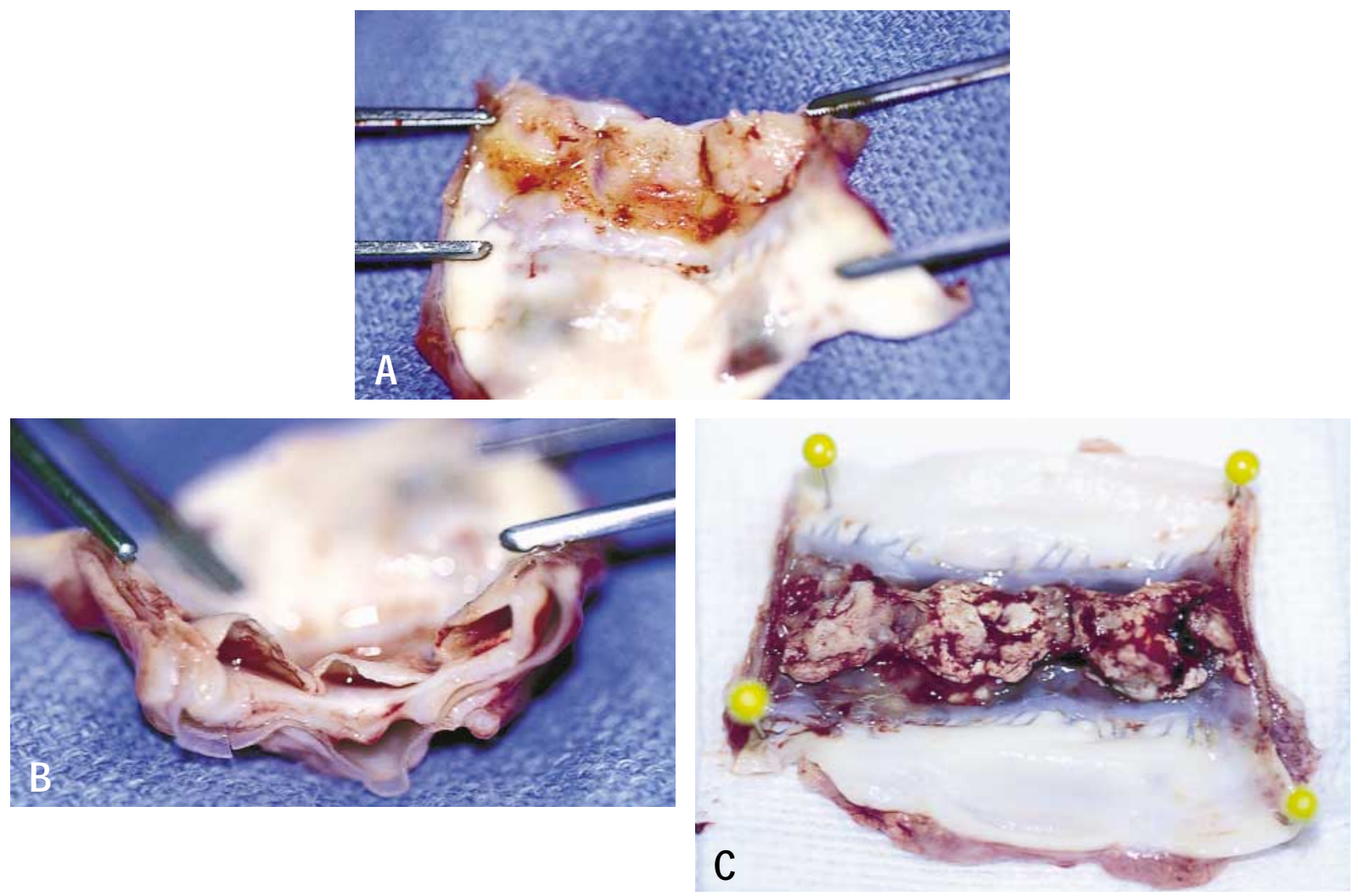

Fig 4. A, Gross appearance of tissue-engineered seeded conduit 24 weeks in vivo (proximal view). B, Gross appearance of tissue-engineered seeded conduit 24 weeks in vivo (distal view). Clear separation of all 3 leaflets from the conduit wall is shown. C, Gross appearance of tissue-engineered unseeded conduit 4 weeks in vivo (proximal view).

ences toward the development of biologic substitutes that restore, maintain, or improve tissue function. ${ }^{9}$ The principal concept is the use of a scaffold material preformed in the shape of the desired organ or structure on which to deliver viable cellular tissue components. Theoretically, the scaffold will provide a temporary biomechanical profile for the replacement tissue until the cells produce their own extracellular matrix. The structural integrity and biomechanical profile for the newly developed tissue structure ultimately depends on this matrix formation. During this process of tissue formation, the scaffold would gradually degrade and resorb and be replaced by the new tissue, eventually leaving no foreign scaffold material within the replaced tissue (Fig 1). ${ }^{10,11}$

In previous experiments we used a combination of PGA and PLA as the scaffold material for the creation of single pulmonary valve leaflets and pulmonary artery segments. ${ }^{1,2}$ Despite the rapid degradation time (approximately 6 weeks), this polymer has several drawbacks. The tissue constructs formed on PGA, with or without PLA, was stiff and consequently less desirable for the creation of a 3-leaflet heart valve.

As an alternative polymer scaffold for tissue engineering, this study evaluated PHO. These are naturally occurring thermoplastic polymers, which are biocompatible, resorbable, and extremely flexible and induce only a minimal inflammatory response. ${ }^{12}$ In recent experiments of our laboratory, infrarenal aortic segments were replaced by using a seeded copolymer of PGA and nonporous PHO. ${ }^{13}$ The favorable results of this study led us to use the same concept for the creation of a 3-leaflet conduit. Experiments with the use of the same copolymer concept on leaflets (PGA-PHOPGA copolymer) failed because of thrombus formation and lack of good tissue formation on the tissue-engineered leaflets. In an attempt to capitalize on the flexibility and strength of PHO while increasing cell attachment and ingrowth, we used salt leaching to create a porous polymer and to thereby potentially increase cell attachment and ingrowth. 

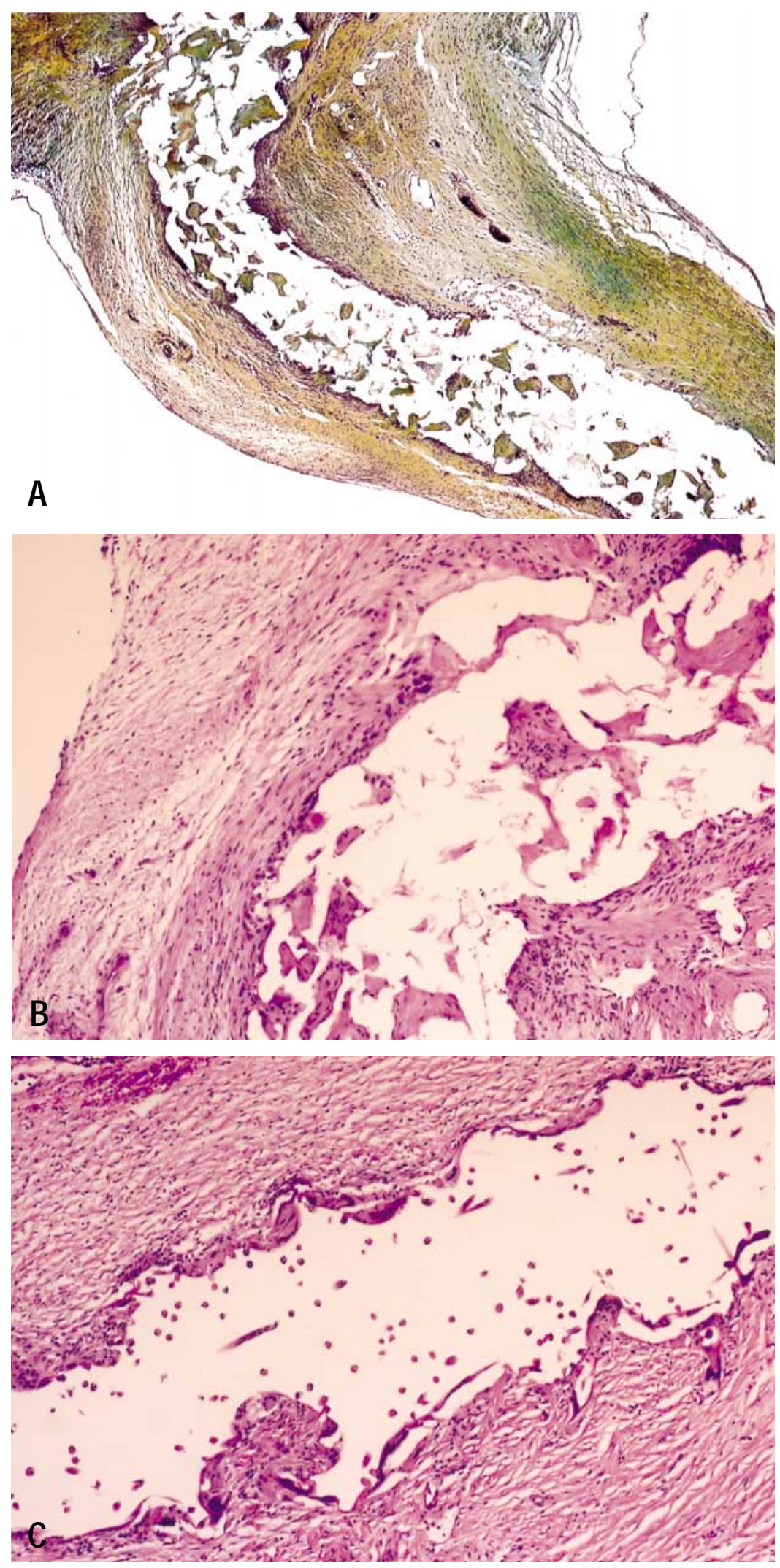

Fig 5. A, Proximal leaflet (16 weeks in vivo) with stain highlighting extracellular matrix elements showing presence of collagen (yellow) and proteoglycans (green). Central space in each photograph represents polymer dissolved during histology. B, Proximal leaflet (16 weeks in vivo) showing fibrous encapsulation ingrowth and absence of thrombus. C, Conduit wall (16 weeks in vivo), with fibrous encapsulation and ingrowth of tissue replacing parts of the polymer. (A, Movat pentachrome stain, magnification $\times 40$; $\mathbf{B}$ and $\mathbf{C}$, hematoxylin and eosin stain, magnification $\times 100$.) 
DNA content of conduit in $\boldsymbol{x}$ of native pulmonary artery

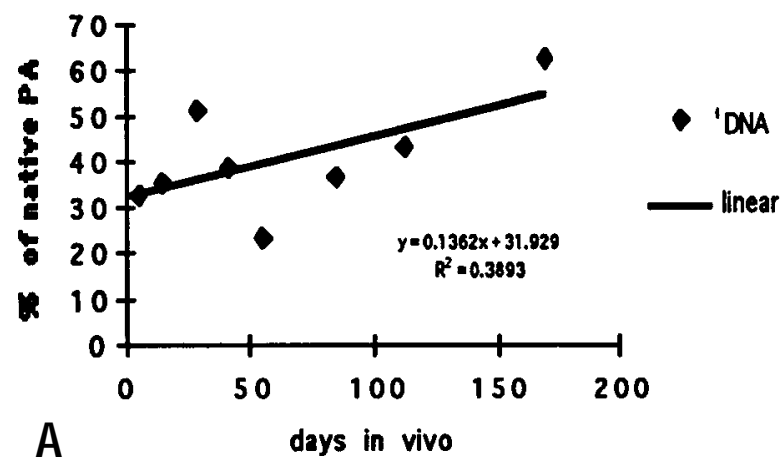

Elastin content of conduit in $\%$ of native
pulmonary artery

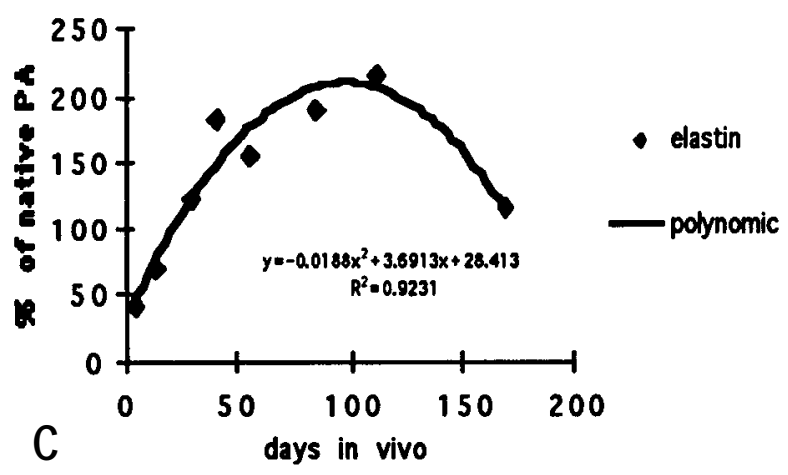

4-OHP content of conduit in $\%$ of native pulmonary artery

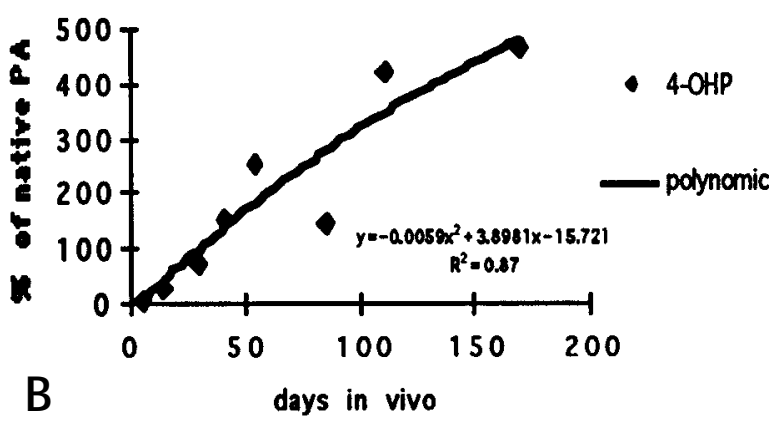

\section{Proteoglycan/glycosaminoglycan content of conduit in $\%$ of native pulmonary artery}

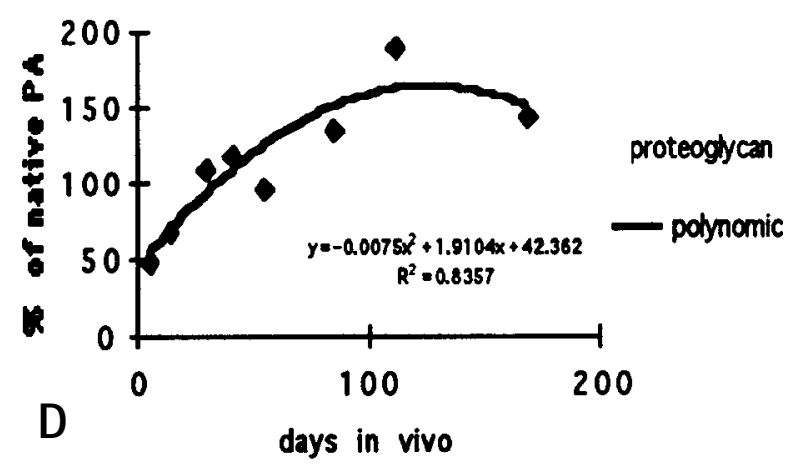

Fig 6. A, DNA content of conduit in percentage of native pulmonary artery. B, 4-Hydroxyproline content of conduit in percentage of native pulmonary artery. $\mathbf{C}$, Elastin content of conduit in percentage of native pulmonary artery. D, Proteoglycan-glycosaminoglycan content of conduit in percentage of native pulmonary artery.

The histologic examination of this study revealed a uniform, organized, fibrous tissue incorporating the conduit wall with an internal endothelial cell lining. Staining showed large amounts of extracellular matrix, including collagen and proteoglycans, in the conduit wall. The biochemical assay for extracellular matrix detected concentrations of collagen, proteoglycans-glycosaminoglycans, and elastin, which exceeded the native pulmonary artery significantly. The leaflets showed a similar histologic pattern with less tissue maturity than the conduit wall but an organized fibrous tissue pattern, with flattened cells corresponding to endothelial cells proximally. In addition, abundant collagen and proteoglycans were present. The explanation for the discrepancy between the elastin assay and the absence of stainable elastin on histologic study is not clear, but it may be due to greater dispersion of elastin in the tissue-engineered conduit than in the native artery.
There was no increase in diameter or length of our constructs over the observed time period. This lack of growth is most likely related to the long degradation time of the PHO. Although no PGA could be detected histologically after 8 weeks in vivo, the PHO used for the conduit wall and the leaflets persisted. The degradation of both polymers, PHO and PGA, is known to occur by hydrolysis. ${ }^{12}$ Although this hydrolysis is known to take 6 to 8 weeks for PGA, we now know from recent publications that the degradation and resorption of plain PHO film exceeds 52 weeks. ${ }^{12}$ The hydrolysis is theoretically determined by surface area. Unpublished results from our laboratory, however, have shown that although salt leaching increases the surface area of PHO, the rate of degradation of PHO is not altered (Stock and associates, unpublished results). On the basis of these findings, we believe that the ideal polymer for the creation of a 3-leaflet conduit should 
Table I. Characteristics of an ideal valve substitute

No inflammatory and/or foreign body response

No immunologic response $\rightarrow$ autologous tissue

Viable structure with autorepair potential and life-long durability

Unlimited supply

Antithrombogenic surface $\rightarrow$ no anticoagulation

Growth potential

Individual custom-made manufacturing

degrade and resorb faster than the PHO used in these studies.

Active research to alter the surface of PHO by using different manufacturing techniques to improve the degradation time and evaluation of other polyhydroxyalkanoates (Metabolix Inc) are currently underway at this institution, and we are currently attempting to improve the scaffold design by use of techniques such as 3-dimensional printing and injection molding techniques. ${ }^{14}$

In addition to polymer- and scaffold design-related limitations, several cell biology issues remain to be clarified. In this and prior experiments we used vascular cells derived from carotid artery explants. It is unlikely that this concept will be applicable to clinical situations, and therefore we are currently investigating the feasibility of using the peripheral vein as the source of donor cells. Mesenchymal stem cells and circulating bone marrow-derived endothelial cells ${ }^{15,16}$ may represent an additional source of cells. A critical issue in tissue engineering is whether the cell populations in and on the constructs are derived from the cells that were seeded on the leaflets and conduit wall or originate from the proximal and distal native pulmonary artery or from circulating pluripotent stem cells. However, reliable celllabeling techniques for long-term implantations were not available when these studies were undertaken. A recent publication by Fox and colleagues ${ }^{17}$ evaluated for the first time optimal labeling conditions for endothelial cells, yielding greatest fluorescence over time without adversely affecting cell viability. Finally, the production and degradation of extracellular components is an important process in the development and modeling of tissue-engineered structures. In this study biochemical assays revealed significantly increased values for collagen, elastin, and proteoglycan-glycosaminoglycans contents, indicating either an increased matrix deposition, a decreased matrix degradation, or both. Normal matrix protein turnover is mediated by a family of enzymes, the matrix metalloproteinases (MMPs), and their natural inhibitors, the tissue inhibitors of metalloproteinases (TIMPs). The balance of MMP and TIMP activity modulates extracellular matrix remodeling. ${ }^{18,19}$

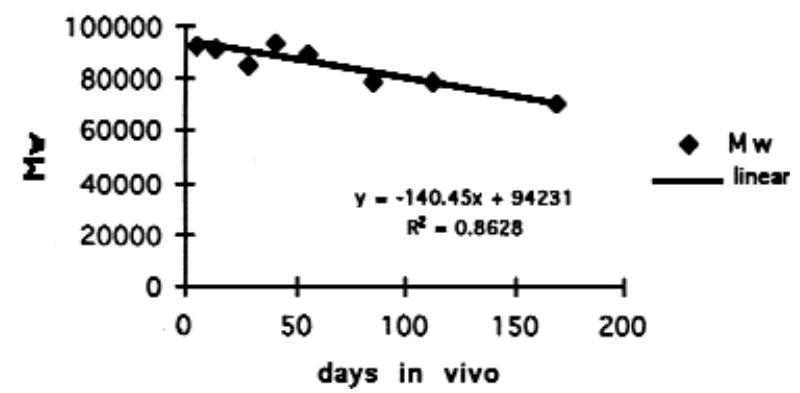

Fig 7. Molecular weight loss of porous PHO material.

We are currently investigating the activity of MMPs and TIMPs in our tissue-engineered constructs to achieve a better understanding of the complex process of tissue formation and remodeling.

\section{Conclusion}

In this study we have fabricated a 3-leaflet heart valve that is able to function for up to 6 months in the pulmonary artery circulation by using tissue-engineering techniques. The porous PHO in the leaflets appeared more favorable to appropriate tissue formation than the film in the conduit wall. However, further work is needed to optimize the scaffold configuration, polymer resorption rate, cell harvesting, and culture conditions to promote the formation of tissue with the appropriate amounts and distribution of matrix proteins. Further advances in polymer sciences, manufacturing techniques, and cell biology offer the potential for improvement, and we are optimistic that the creation of tissue-engineered heart valves will become possible in the long run.

\section{REFERENCES}

1. Shinoka T, Breuer C, Tanel R, et al. Tissue engineering heart valves: valve leaflet replacement study in a lamb model. Ann Thorac Surg 1995;60:S513-6.

2. Shinoka T, Shum-Tim D, Ma P, et al. Creation of a viable pulmonary artery autograft through tissue engineering. J Thorac Cardiovasc Surg 1998;115:536-46.

3. Shinoka T, Ma P, Shum-Tim D, et al. Tissue-engineered heart valves: autologous valve leaflet replacement study in a lamb model. Circulation 1996;94(Suppl):II-164-8.

4. Mooney DJ, Breuer C, McNamara K, et al. Fabricating tubular devices from polymers of lactic and glycolic acid for tissue engineering. Tissue Eng 1995;1:107-18.

5. Bergman I, Loxley R. Two improved and simplified methods for the spectrophotometric determination of hydroxyproline. Anal Chem 1963;35:1961-5.

6. Schoen FJ, Levy RJ. Tissue heart valves: current challenges and future research perspectives. J Biomed Mater Res 1999;47:439-65.

7. Mayer JE Jr. Uses of homograft conduits for right ventricle to pulmonary artery connections in the neonatal period. Semin Thorac Cardiovasc Surg 1995;7:130-2. 
8. Harken DW, Curtis LE. Heart surgery: legend and a long look. Am J Cardiol 1967;19:393-400.

9. Skalak R, Fox CF. Tissue engineering. Ann Biomed Eng 1991;19:529-33.

10. Vacanti JP. Beyond transplantation. Third Annual Samuel Jason Mixter Lecture. Arch Surg 1988;123:545-9.

11. Langer R, Vacanti JP. Tissue engineering science. 1993;260:920-6.

12. Williams SF, Martin DP, Horowitz DM, Peoples OP. PHA applications: addressing the price performance issue. I. Tissue engineering. Int J Biol Macromol 1999;25:111-21.

13. Shum-Tim D, Stock U, Hrkach J, et al. Tissue engineering of autologous aorta using a new biodegradable polymer. Ann Thorac Surg 1999;68:2298-305.

14. Sachs E, Cima M, Williams P, et al. 3-Dimensional printingrapid tooling and prototypes directly from a CAD model. J Eng Industry Trans ASME 1992;114:481-8.

15. Pittenger MF, Mackay AM, Beck SC, et al. Multilineage potential of adult human mesenchymal stem cells. Science 1999;384:143-7.

16. Shi Q, Rafii S, Hong-De Wu M, et al. Evidence for circulating bone marrow-derived endothelial cells. Blood 1998;92:362-7.

17. Fox D, Kouris GJ, Blumofe KA, et al. Optimizing fluorescent labeling of endothelial cells for tracking during long-term studies of autologous transplantation. J Surg Res 1999;86:9-16.

18. Moses M, Shing Y. Production of matrix metalloproteinases and metalloproteinase inhibitor by Swarm rat chondrosarkoma. Biochem Biophys Res Commun 1994;199:418-24.

19. Braunhut SJ, Moses M. Retinoids modulate endothelial cell production of matrix-degrading proteases and TIMPs. J Biol Chem 1994;269:13472-9.

\section{Discussion}

Dr Alain F. Carpentier (Paris, France). I congratulate the authors on a very interesting study. I published an experimental study similar to yours, entitled "Collagen-Derived Heart Valves," in The Journal of Thoracic and Cardiovascular Surgery in 1971 (1971;62:707-13). I wish to share with you what we have learned from this study in the hope that it may be helpful to you.
First, if you make a valved conduit without Valsalva sinuses, a fibrous retraction of the cusps takes place after a few months, whatever the type of tissue-engineered valve. With a longer follow-up, you may be confronted with this complication. If you see it, rather than blaming the valve, I suggest that you make a new conduit with Valsalva sinuses, which will give your tissue-engineered valve the best chance of success.

With regard to the cultivated cells you used, it was not clear whether you used fibroblasts, smooth cells, or endothelial cells. Also, what was the proportion of these different cells and their position in the valve tissue?

Dr Stock. We undertook these experiments as an initial feasibility study to test the concept of tissue engineering of a 3-leaflet heart valve. We are well aware that a normal semilunar valve has the sinuses of Valsalva, and in this case we do not have any sinuses. We are now working with different manufacturers with 3-dimensional printing and injection molding techniques, to make a semilunar valve that really is like an identical copy of a human or an animal heart valve; we then include the sinus.

Dr Carpentier. Very simply, are these cells endothelial cells or are they fibroblast cells?

Dr Stock. We have endothelial cells, and we have mixed cells of smooth muscle cells and fibroblasts. We have two populations.

Dr Carpentier. If you have a mixed population, I am sure you are aware that the fibroblasts may develop at the expense of the endothelial cells. Would it not be better to have only endothelial cells?

Dr Stock. We cultured them separately, and therefore we have one dish with endothelial cells and one dish with smooth muscle cells and fibroblasts. This is a complete separation.

Dr Carpentier. Thank you. I do hope that with the more accurate techniques and materials that we have today, you are going to overcome the problem of tissue retraction that we encountered in the past. 